\title{
Epigenetic modification is involved in aberrant expression of class III B-tubulin, TUBB3, in ovarian cancer cells
}

\author{
NAOKO IZUTSU ${ }^{1,2}$, CHIHAYA MAESAWA ${ }^{1}$, MASAHIKO SHIBAZAKI ${ }^{1}$, HIROKI OIKAWA ${ }^{1}$, \\ TADAHIRO SHOJI $^{2}$, TORU SUGIYAMA ${ }^{2}$ and TOMOYUKI MASUDA ${ }^{1}$ \\ Departments of ${ }^{1}$ Pathology and ${ }^{2}$ Obstetrics and Gynecology, School of Medicine, \\ Iwate Medical University, Uchimaru 19-1, Morioka, Iwate 020-8505, Japan
}

Received December 24, 2007; Accepted February 5, 2008

\begin{abstract}
Aberrant expression of class III B-tubulin, TUBB3, has been reported to be one of the important mechanisms responsible for taxane resistance in diverse human malignancies. We investigated aberrant TUBB3 expression and its epigenetic modification in 66 primary tumors and 3 cell lines (OVCAR-3, JHOC-5 and JHOC-8) of ovarian cancers. Overexpression of TUBB3 protein was observed in $56(85 \%)$ of the 66 ovarian cancers, and was significantly associated with aggressive tumor behavior (advanced stage, presence of ascites, suboptimal cytoreduction at surgery and presence of lymph node metastasis) $(\mathrm{P}<0.05)$. Responses to treatment with a demethylating agent (5-aza-2'-deoxycytidine, 5Aza-CdR) and a histone deacetylase inhibitor (4-phenylbutyric acid, PBA) differed among the ovarian cancer cell lines. In 2 cell lines with weak expression of TUBB3 protein (OVCAR-3 and JHOC-8), TUBB3 induction was independently induced by treatment with 5-Aza-CdR (JHOC-8) or PBA (OVCAR-3), while neither agent markedly altered TUBB3 mRNA/protein expression in a strongly TUBB3expressing cell line (JHOC-5). A CpG island within intron 1 was hypermethylated in 1 cell line (JHOC-8) that expressed TUBB3 weakly and required 5-Aza-CdR treatment for gene expression. A CpG island of another cell line showing faint expression of TUBB3 protein (OVCAR-3), in which a significant gain of TUBB3 expression was induced by treatment with PBA but not with 5-Aza-CdR, was hypomethylated, similarly to a cell line (JHOC-5) showing constitutive expression of TUBB3. We evaluated methylation status in this region in 14 primary tumors using methylation-
\end{abstract}

Correspondence to: Dr Chihaya Maesawa, Department of Pathology, Iwate Medical University, Uchimaru 19-1, Morioka, Iwate 020-8505, Japan

E-mail: chihaya@iwate-med.ac.jp

Key words: TUBB3, epigenetics, taxane, drug resistance, ovarian cancer specific PCR, but there was no significant relationship with TUBB3 immunoreactivity. These findings suggest that aberrant expression of TUBB3 protein might be associated with aggressive behavior of ovarian cancers, and that epigenetic modulation (DNA methylation and chromatin acetylation) might be partly involved in TUBB3 expression.

\section{Introduction}

Ovarian cancer represents the fifth leading cause of cancer death for women in the United States (1). More than $70 \%$ of cases are at an advanced stage at diagnosis. Despite significant advances in surgery and chemotherapy over the last few decades, the overall 5-year survival rate of patients with advanced ovarian cancer remains quite low (1-3). The current standard treatment of epithelial ovarian cancer involves primary optimal debulking surgery followed by platinum-taxane-based chemotherapy $(2,3)$. Although this treatment yields a high objective response rate, the large majority of patients ultimately suffer relapse of the malignancy $(2,3)$, possibly due to the development of resistance to platinum-based agents and taxanes.

The molecular mechanisms of tumor resistance to platinum agents have been shown to include mutation of $\mathrm{p} 53$, and alterations of the DNA mismatch repair system (4-6), whereas much attention has recently been focused on the role of tubulin alterations in supporting resistance to taxanes (7). In particular, point mutations of the tubulin genes have been associated with taxane resistance in in vitro models $(8,9)$, although no unequivocal data are currently available (10-12). Moreover, overexpression of selective $\beta$-tubulin isotypes has been advocated as an additional mechanism involved in paclitaxel resistance (13-21). Six B-tubulin isotypes have been identified in mammalian cells, encoded by different genes and with distinct patterns of tissue expression (22). The class III B-tubulin, TUBB3, is a well known neuronal tubulin that is expressed in the earliest phase of neural differentiation and is neuron-associated (22). TUBB3 has been hypothesized to counteract the suppression of microtubule dynamicity by paclitaxel through enhancement of microtubule instability or, alternatively, reduction of the microtubule polymerization rate (13-21). 
Recent reports have documented that ovarian cancer patients showing overexpression of TUBB3 protein have a worse prognosis $(21,23,24)$, as is the case for other types of malignancy $(25,26)$, although the molecular mechanisms of TUBB3 protein overexpression have not been fully elucidated. It is well known that epigenetic alterations (DNA methylation and chromatin modification) are associated with overexpression of the multidrug resistance gene (ABCB1: ATP-binding cassette, sub-family B, MDR/TAP, member 1), resulting in tumor resistance to platinum agents $(27,28)$. However, the epigenetic mechanisms related to aberrant TUBB3 protein expression have remained unclear. In the present study, we focused on the epigenetic mechanisms responsible for regulation of TUBB3 protein overexpression in ovarian cancer cell lines.

\section{Materials and methods}

Cell lines and specimens. Three cell lines, OVCAR-3 (Institute of Development, Aging and Cancer, Tohoku University, Sendai, Japan), JHOC-5 (Riken Cell Bank; RCB, Tsukuba, Japan) and JHOC-8 (RCB), were used. They were maintained under their recommended conditions.

We also immunohistochemically examined 66 patients with ovarian cancer and 10 patients with benign cystic lesions of the ovary, treated between 1998 and 2005 at the Department of Obstetrics and Gynecology, School of Medicine, Iwate Medical University, Morioka, Japan. Surgical specimens were fixed in $10 \%$ buffered formalin solution and embedded in paraffin wax, and two or more blocks were prepared for immunohistochemistry. Fourteen of the 66 ovarian cancer patients were also examined by methylationspecific PCR (MSP). All patients underwent debulking surgery included surgical removal of the tumor masses along with total abdominal hysterectomy, adnexectomy, radical omentectomy, and appendectomy plus additional surgery (diaphragm stripping, intestinal resection, posterior pelvic exenteration and low rectal anastomosis) if necessary. After the surgery, they underwent chemotherapy using a combination of platinum agents, paclitaxel, and/or irinotecan. Fortyeight of the patients underwent paclitaxel-based chemotherapy. Permission for the study was obtained from the Institutional Review Board (School of Medicine, Iwate Medical University) and written consent was obtained from all patients before surgery.

Immunohistochemistry. Four-micrometer-thick sections were cut from formalin-fixed, paraffin-embedded samples, and stained with hematoxylin and eosin. Serial sections were stained using the avidin-biotin system and antigen retrieval methods on a Ventana automated immunostainer with the Ventana immunohistochemistry detection system (Ventana Medical Systems, Tucson, AZ, USA), in accordance with the manufacturer's manual. The primary antibody used for TUBB3 immunostaining was a mouse monoclonal anti-class III B-tubulin antibody (diluted 1:500; Covance, Princeton, NJ, USA). Quantitative comparative analysis of immunohistochemical staining was carried out in each case. The extent of immunohistochemical reactivity was estimated by light microscopy and graded according to the number of immunoreactive cells (proportion score) and staining intensity (intensity score). In brief, proportion scores were graded as follows: 0, no immunoreactive cells evident; 1 , proportion of immunoreactive cells $<1 \%$; $2,<10 \%$; $3,<1 / 3$; $4,<2 / 3$; and $5,>2 / 3$. Intensity scores were graded as follows: 0 , no immunoreactivity; 1 , staining intensity weak; 2 , intermediate; and 3, strong. The final estimation of immunoreactivity was assigned as negative/faint if the sum of the proportion and intensity scores ranged from 0 to 2 , medium if the sum ranged from 3 to 5 , and strong if the sum ranged from 6 to 8 .

5-aza-2'-deoxycytidine (5-Aza-CdR) and 4-phenylbutyric acid (PBA) treatment. To evaluate the expression of TUBB3 protein, we treated ovarian cancer cell lines with a demethylation agent (5-Aza-dC, $3 \mu \mathrm{M})$ and/or a histone deacetylase (HDAC) inhibitor (PBA, $3 \mathrm{mM}$ ) as described previously (29). Cells were seeded at $3 \times 10^{5}$ cells per $60-\mathrm{mm}$ Petri dish $24 \mathrm{~h}$ prior to the treatment. 5-Aza-CdR was removed after $24 \mathrm{~h}$, while PBA was administered continuously by replacing the medium containing PBA every $24 \mathrm{~h}$ for 5 days. After the treatment, total RNA was obtained for real-time quantitative RT-PCR for TUBB3 mRNA and Western blotting for TUBB3 protein, respectively.

Real-time quantitative PCR assay. In the real-time quantitative (RQ)-RT-PCR assay, primers and a fluorogenic probe were designed with Primer Express software (ABI): TUBB3-115F (GGG CCA AGT TCT GGG AAG TC), TUBB3-183R (AGT CGC CCA CGT AGT TGC C) and probe (TUBB3-140T, FAM-TGA TGA GCA TGG CAT CCA TCG ACC CCA GTAMRA). The RQ-RT-PCR assay was done using an ABI PRISM 7500 Sequence Detector (Applied Biosystems; ABI, Foster City, CA, USA). The reaction mix contained $50 \mathrm{ng}$ of cDNA, $200 \mathrm{nmol} / 1$ each primer, $5 \mu \mathrm{M}$ probe, and $25 \mu \mathrm{l}$ of TaqMan Universal PCR Master Mix (ABI) in a final volume of $50 \mu \mathrm{l}$. The cDNA was subjected to 50 cycles of a two-step PCR consisting of a 15-sec denaturation step at $95^{\circ} \mathrm{C}$ and a $1-$ min combined annealing/extension step at $60^{\circ} \mathrm{C}$. Plasmids were diluted in a precise series, ranging from $5 \mathrm{pg}$ to $0.005 \mathrm{fg}\left(2 \times 10^{6}\right.$ to 2 copies $)$. For normalization of each target in the samples, the copy number of glyceraldehyde-3-phosphate dehydrogenase (GAPDH) was used as an internal control as described previously (30).

Western blotting. The cell suspension was centrifuged, and after removal of the supernatant the cell pellet was dissolved in 1.0\% NP-40 lysis buffer [50 mM HEPES (pH 7.5)/ $1 \mathrm{mM}$ EDTA/150 mM NaCl/2.5 mM EGTA/1.0\% NP-40]. Cell samples containing equal amounts of protein were mixed with 6-fold concentrated loading dye, heated for $4 \mathrm{~min}$ at $95^{\circ} \mathrm{C}$, and subjected to SDS-PAGE on a $7.5 \%$ polyacrylamide gel (Bio-Rad Laboratories, Hercules, CA, USA). The proteins were then transferred to a PVDF (polyvinylidene difluoride) membrane (Amersham Biosciences, Buckinghamshire, UK) by electroblotting. Primary antibodies against TUBB3 (Antiß-Tubulin III; Sigma Chemical Co. Ltd., St. Louis, MO, USA) and glyceraldehyde-3-phosphate dehydrogenase (antiGAPDH; Upstate Biotech, Lake Placid, NY, USA) were diluted 1:1,000 in 0.05\% Tween-20 PBS. The membrane was 
incubated for $1 \mathrm{~h}$ at room temperature and washed. For the second antibody, anti-mouse IgG or anti-rabbit IgG (Amersham Biosciences, NJ, USA) was diluted 1:5,000 in blocking buffer. The membrane was then incubated for $45 \mathrm{~min}$ at room temperature and washed. Signals were detected with ECL Plus (Amersham Biosciences) and ChemiDoc XRS (Bio-Rad). The intensity of the detected signals was measured by 1-D analysis software (Quantity One, Bio-Rad). For normalization of the target, GAPDH was used as an internal control.

Bisulfite genome sequencing. DNAs obtained from the cell lines were subjected to bisulfite modification and examined for the methylation status of $103 \mathrm{CpG}$ sites around the transcriptional start sites. Bisulfite modification was done using a Methylamp ${ }^{\mathrm{TM}}$ DNA Modification Kit (Epigenetek Inc., Broadway, NY, USA). Modified DNA was PCRamplified using 3 primer sets: TUBB3-biF2153 (GTT TAG TGT TGG GGG ATT TTT GGT TGT)/TUBB3-biR2586 (CTA AAC ACA TCC CTT TAT TAC AAA TT), TUBB3biF5097 (GGA TAT AGG ATG TTG TTT TAT TGT)/ TUBB3-biR5411 (AAA CTA AAA CCT CCA AAC CCC TCC), and TUBB3-biF5388 (GGA GGG GTT TGG AGG TTT TAG TTT)/TUBB3-biR5611 (ACC AAA CCT CCT CTC ATA ACC A). The final reaction volume for all PCRs was $50 \mu \mathrm{l}$, and the mixture contained $0.2 \mu \mathrm{M}$ each primer, $1 \mathrm{mM} \mathrm{MgCl}{ }_{2}, 0.2 \mathrm{mM}$ each dNTP, $2.5 \mathrm{U}$ of Platinum Taq DNA polymerase (Invitrogen, Carlsbad, CA, USA) and $30 \mu 1$ of distilled water. Amplified PCR products were analyzed by electrophoresis on a $2 \%$ agarose gel. PCR products were purified with a QIAquick Gel Extraction Kit (Qiagen, Hilden, Germany). The PCR fragments were ligated to pGEM-T Easy Vectors (Promega Co., Madison, WI, USA) and transformed into DH5 $\alpha$ competent cells (Toyobo Co., Ltd., Osaka, Japan). Ten subcloned colonies were chosen at random from each ovarian cancer cell line. Plasmid DNA was purified by a PI-200 DNA automatic isolation system (Kurabo, Osaka, Japan). Cycle sequencing was done using a BigDye Terminator v3.1 Cycle Sequencing Kit (ABI) and an ABI Prism 3100 DNA Sequencer (ABI).

Methylation-specific PCR (MSP). For analysis of the methylation status of the surgical specimens, we used the MSP method as described previously (31). Briefly, DNAs were treated with a CpGenome ${ }^{\mathrm{TM}}$ DNA Modification Kit (Intergen, Purchase, NY, USA). Two sets of primers were designed to specifically amplify either the methylated (M) (TUBB3 MF5241: GTA TCG TTT TTT TGC GGT TGT GGG GCG GGG/TUBB3 MR5382: CCG AAT ACG ATT CCC GCC CGA CCC GCA ACT) or unmethylated (U) (TUBB3 UMF5241: GTA TTG TTT TTT TGT GGT TGT GGG GTG GGG/TUBB3 UMR5382: CCA AAT ACA ATT CCC ACC CAA CCC ACA ACT) bisulfite-modified sequence, by using the MethPrimer program (http://www. methdb.de). PCR conditions and negative controls have been described elsewhere (31).

Chromatin immunoprecipitation (ChIP) assay. The ChIP assay with acetyl-histone-H3 (Upstate Biotech) and -H4 (Upstate Biotech), and anti-HDAC1 (clone 2E10, Upstate
Biotech) anti-MeCP2 (N-17, sc-5755, Santa Cruz Biotechnology, Santa Cruz, CA, USA) was performed using the Shearing-ChIP Kit ${ }^{\mathrm{TM}}$ (Diagenode, Liège, Belgium) and OneDay ChIP Kit ${ }^{\mathrm{TM}}$ (Diagenode) in accordance with the manufacturer's instructions. The RQ-PCR assay was used to analyze ChIP DNA with the ABI Prism 7500 sequence detector $(A B I)$. Primers and fluorogenic probes for the TUBB3 and GAPDH promoters were designed with Primer Express software (ABI): TUBB3-313F (GCA CCA AGG ACA GCG CC), TUBB3-563R (GCA ACA AGA GCC AAA CTC CAT C), TUBB3-313CProbe (FAM-CTG GCC TCG AAC GCC GGG TT-TAMRA), GAPDH CF (5'-CTA GTG TCC TGC TGC CCA C-3'), GAPDH CR (5'-AGG TCT TGA GGC CTG AGC TAC-3'), GAPDH Cprobe (5'-FAMAGC GGT TTT ACG GGC-TAMURA-3'). The PCR conditions and reaction mixtures employed have been described previously (32). A standard curve method was used for quantitative evaluation of the ChIP DNAs.

Statistical analysis. Correlations between TUBB3 immunoreactivity and clinicopathological data were analyzed by non-parametric Mann-Whitney U test or Fisher's exact test. Correlation between TUBB3 protein expression and methylation status at the TUBB3 promoter region was analyzed by Fisher's exact test. All data are presented as the mean \pm SE. To correlate the results of TUBB3 protein immunostaining with overall survival of patients with ovarian cancers, Kaplan-Meier survival analysis and log-rank test were performed. The level of significance was set at $\mathrm{P}<0.05$.

\section{Results}

Immunohistochemistry for TUBB3 protein in primary ovarian cancers. Positive immunoreactivity for TUBB3 protein was restricted only to nerve cells in normal tissues of the 10 patients with benign ovarian cyst (Fig. 1A). The likely origin of ovarian surface epithelial-stromal tumors is the mesothelial surface lining of the ovaries and/or invaginations of this lining into the superficial ovarian cortex that form inclusion cysts. Benign cysts and surface lining cells were negative in all 10 patients with non-neoplastic cystic lesions of the ovary (Fig. 1B).

Immunoreactivity for TUBB3 protein varied among ovarian cancers (Fig. 1C-E). Sublocalization of TUBB3 protein was positive in the cytoplasm. Table I summarizes the relationship between TUBB3 immunoreactivity and clinicopathological variables of cases in the whole series. Positive TUBB3-immunoreactivity $(n=56,85 \%$ : medium, 30; and strong, 26 vs. negative/faint, 10) was frequently observed in patients with advanced disease, ascites at presentation, suboptimal cytoreduction at surgery, and presence of lymph node metastasis than in those without these factors. There was no significant relationship with histological type. Evaluation of the relationship between TUBB3 immunoreactivity and clinical responses of patients undergoing paclitaxel-based chemotherapy revealed no significant correlation. Follow-up data were available for all patients. After a median follow-up of 46 months (range, 2-109 months), progression and death due to disease were observed in $8(12.1 \%)$ and $17(25.8 \%)$ 


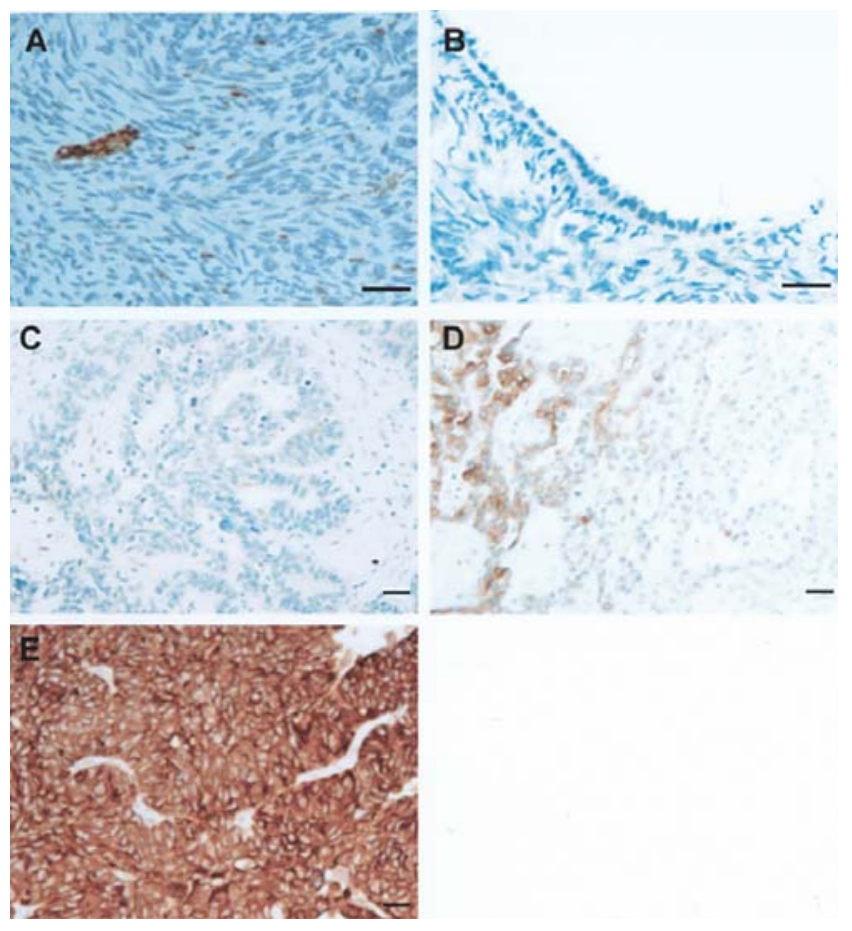

Figure 1. Immunohistochemistry for TUBB3 in ovarian cancer and ovary with benign cystic lesions. (A) Positive immunoreactivity for TUBB3 protein is observed in nerve cells in a patient with benign ovarian cyst. (B) Mesothelial surface lining cells, which are the likely origin of ovarian surface epithelial-stromal tumors, are negative for the TUBB3 protein. (C-E) TUBB3 immunoreaction in primary ovarian cancers. Quantitative comparative analysis (proportion and intensity scores) of immunohistochemical staining for TUBBB3 protein was performed, as described in Materials and methods. Assessments of 3 cases are shown (C, negative; D, medium; E, strong). Bar, $100 \mu \mathrm{m}$.

cases, respectively. The survival curves of the patients revealed no statistically significant differences with regard to the presence or absence of TUBB3 immunoreactivity (Fig. 2).

TUBB3 mRNA/protein expression and 5-Aza-CdR and/or $P B A$ treatment in ovarian cancer cell lines. Expression of TUBB3 was investigated in 3 cell lines established from human ovarian cancers by means of Western blot and RQPCR analyses. TUBB3 protein was strongly expressed in 1 cell line (JHOC-5), whereas the signals of OVCAR-3 and JHOC-8 were faint and relatively weak, respectively (Fig. 3A). Quantitative results for TUBB3 mRNA were compatible with protein expression (Fig. 3B).

Changes in expression of TUBB3 protein by treatments with 5-Aza-CdR and/or PBA differed among the cell lines. The cell line JHOC-5, strongly overexpressing TUBB3, did not exhibit a marked increase in expression of TUBB3 protein (Fig. 4A). In OVCAR-3, a cell line exhibiting faint immunoreactivity for TUBB3 protein, treatment with 5-Aza-CdR increased TUBB3 mRNA/protein expression slightly (Fig. 4), whereas PBA treatment alone induced a marked gain of TUBB3 protein expression in comparison with 5-Aza-CdR treatment. Combination of the 2 agents slightly accelerated the gain of TUBB3 protein expression in comparison with treatment with each agent alone, but did not affect mRNA
Table I. Correlation between TUBB3 immunoreactivity and clinicopathological variables in 66 patients with ovarian cancer.

\begin{tabular}{|c|c|c|c|}
\hline \multirow[b]{2}{*}{ Variable } & \multicolumn{3}{|c|}{ TUBB3 immunoreactivity } \\
\hline & $\begin{array}{c}\text { Positive } \\
(n=56)\end{array}$ & $\begin{array}{l}\text { Negative } \\
(n=10)\end{array}$ & P-value \\
\hline Age (years) & $54.3(21-82)$ & $53.9(43-65)$ & \\
\hline \multicolumn{4}{|l|}{ Stage } \\
\hline I, II & 22 & 9 & 0.003 \\
\hline III, IV & 34 & 1 & \\
\hline \multicolumn{4}{|l|}{ Ascites } \\
\hline No & 8 & 5 & 0.009 \\
\hline Yes & 48 & 5 & \\
\hline \multicolumn{4}{|l|}{ Histology } \\
\hline Serous & 38 & 5 & 0.270 \\
\hline Others & 18 & 5 & \\
\hline \multicolumn{4}{|l|}{ Cytoreduction } \\
\hline Optimal & 31 & 9 & 0.040 \\
\hline Suboptimal & 25 & 1 & \\
\hline \multicolumn{4}{|l|}{ Node status } \\
\hline Positive & 34 & 1 & 0.002 \\
\hline Negative & 14 & 7 & \\
\hline Not done & 8 & 2 & \\
\hline
\end{tabular}

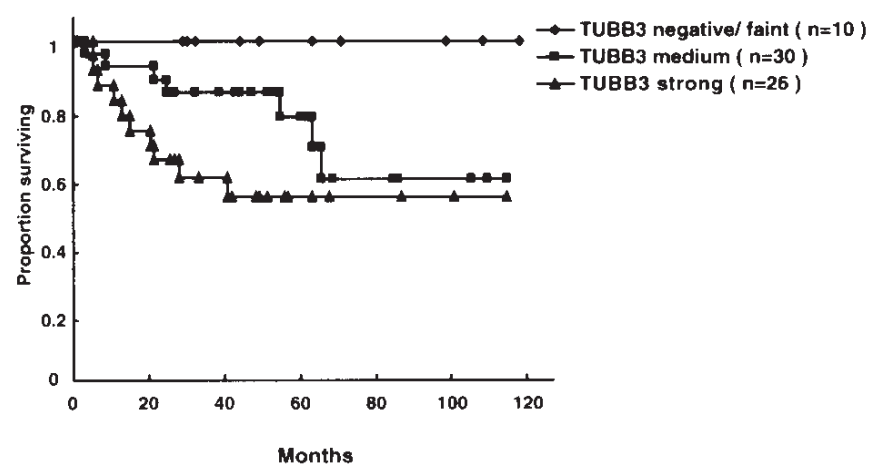

Figure 2. Survival curves for 66 ovarian cancer patients according to TUBB3 immunoreactivity.

expression (Fig. 4). In another cell line, JHOC-8, which shows weak TUBB3 mRNA/protein expression, single treatment with 5-Aza-CdR induced a gain of TUBB3 mRNA/ protein expression, but single treatment with the HDAC inhibitor PBA did not (Fig. 4). Combination of the 2 agents accelerated the gain of TUBB3 mRNA/protein in comparison with 5-Aza-CdR, but the difference was not significant. As 
A

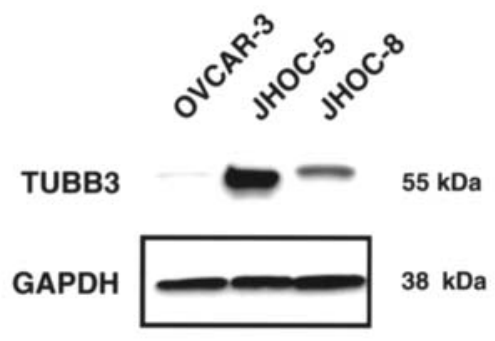

B

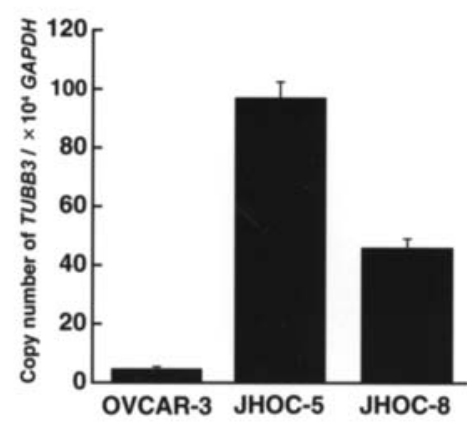

Figure 3. Results of Western blotting and real-time quantitative PCR for TUBB3 mRNA/protein expression in 3 ovarian cancer cell lines. (A) JHOC-5 exhibited overexpression of TUBB3 protein, whereas OVCAR-3 and JHOC-8 exhibited faint and weak expression, respectively. Twenty micrograms of whole-cell lysate were applied to each lane. Equal loading was confirmed by blotting of GAPDH. (B) Quantitative results for TUBB3 mRNA are compatible with the results of protein expression. differences in the epigenetic status of the TUBB3 gene were considered to play a possible role, we further determined the DNA methylation and histone acetylation status of the TUBB3 gene in these cell lines.

DNA methylation and histone acetylation status in ovarian cancer cell lines. Two $\mathrm{CpG}$ islands are located around the transcriptional site of the TUBB3 gene (Fig. 5A). The first $\mathrm{CpG}$ island showed DNA hypomethylation in all ovarian cancer cell lines (Fig. 5B). However, the second $\mathrm{CpG}$ island within intron 1 was heavily methylated in one weakly TUBB3-positive cell line (JHOC-8) in which 5-Aza-CdR treatment was necessary for gain of TUBB3 mRNA/protein expression (Fig. 4). Another cell line (OVCAR-3) exhibiting faint TUBB3-positivity, which did not require the demethylating agent for gain of TUBB3 mRNA/protein expression, exhibited hypomethylation in this region (Fig. 5C).

To assess whether chromatin modifications at the second $\mathrm{CpG}$ island of the TUBB3 gene are associated with mRNA/protein expression, we performed ChIP experiments with antibodies against the acetylated histones, MeCP2 and HDAC1 (Fig. 6). In JHOC-5 and JHOC-8, treatment with PBA did not induce marked quantitative alteration of acetylated $\mathrm{H} 3$ and $\mathrm{H} 4, \mathrm{HDAC} 1$ and $\mathrm{MeCP} 2$. These results were concordant with those of Western blotting and RQPCR assays for TUBB3 mRNA/protein. After treatment with PBA, the histone-H3 and -H4 acetylation in OVCAR-3 was increased 2-3 fold. The ChIP results for HDAC1 and MeCP2

A

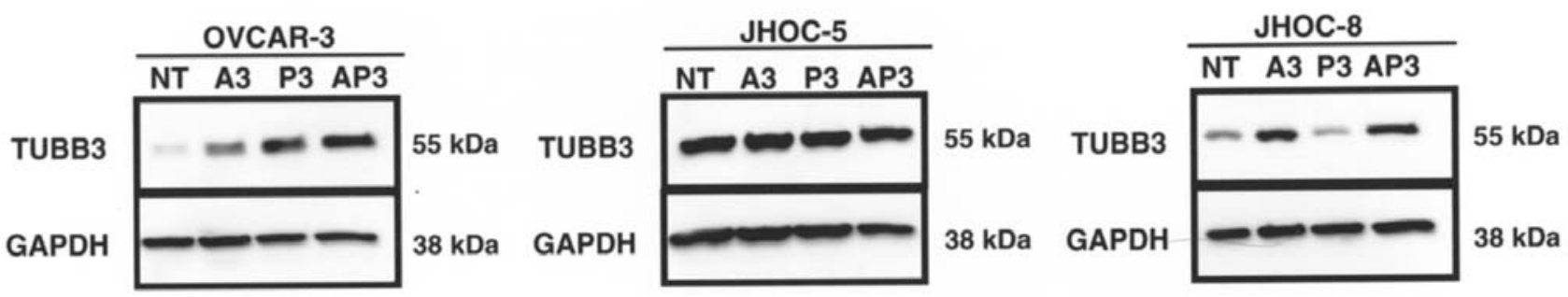

B

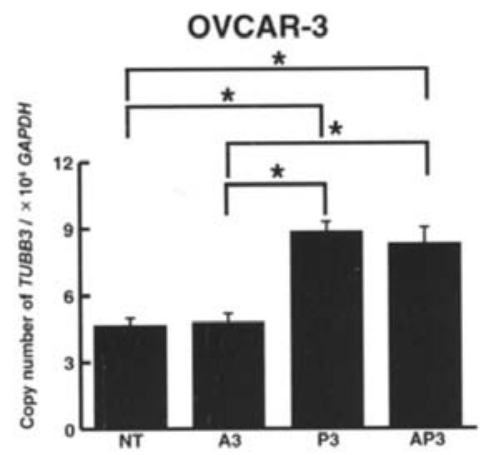

JHOC-5

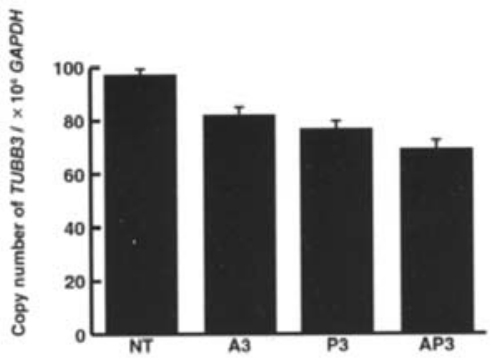

JHOC-8

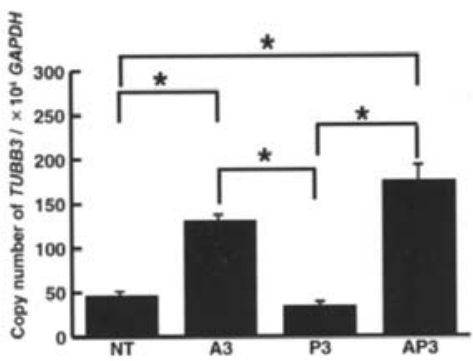

Figure 4. TUBB3 mRNA/protein expression in 3 ovarian cell lines treated with a demethylation agent (A3, $3 \mu \mathrm{M} 5$-Aza-CdR) and a histone deacetylase inhibitor (P3, $3 \mathrm{mM}$ PBA). AP3, combination of $3 \mu \mathrm{M}$ 5-Aza-CdR and $3 \mathrm{mM}$ PBA. NT, untreated control. (A) Western blot analyses for TUBB3 protein indicate that the expression level of TUBB3 is not altered in the strongly TUBB3-overexpressing cell line JHOC-5. In contrast, a cell line exhibiting faint immunoreactivity for TUBB3 protein, OVCAR-3, exhibits a slight increase of TUBB3 protein expression upon single treatment with 5-Aza-CdR, whereas single treatment with PBA induces marked TUBB3 protein expression. In JHOC-8, which is a cell type showing weak TUBB3 mRNA/protein expression, single treatment with 5-Aza-CdR induces a gain of TUBB3 protein expression, whereas single treatment with PBA does not. Twenty micrograms of wholecell lysate were applied to each lane. Equal loading was confirmed by blotting of GAPDH. (B) Quantitative results for TUBB3 mRNA are compatible with those of protein expression. ${ }^{*} \mathrm{P}<0.05$. 
A

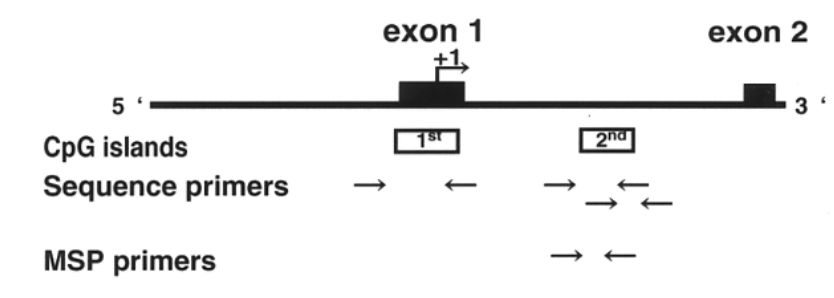

B

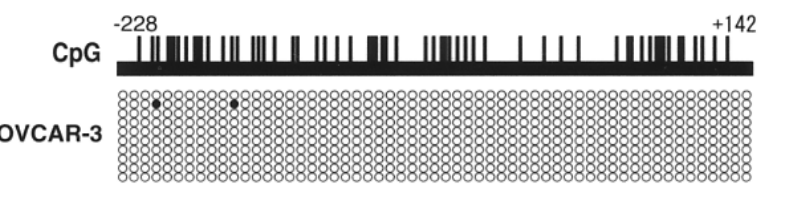

JHOC-5

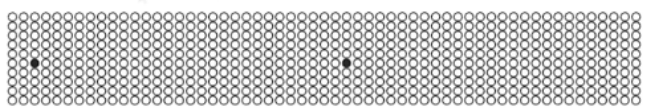

JHOC-8

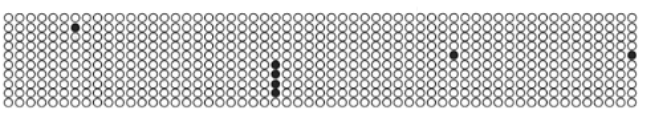

C
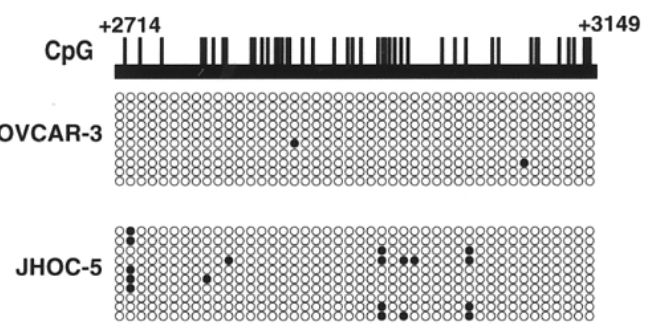

JHOC-8

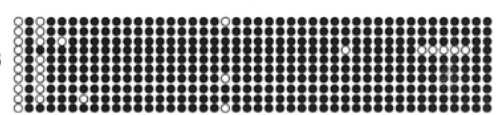

Figure 5. DNA methylation status of two $\mathrm{CpG}$ islands around the transcriptional start site of the TUBB3 gene in 3 ovarian cancer cell lines. (A) Diagram of TUBB3 gene CpG islands (squares). Primers used for bisulfite genome sequencing (Sequence primers) and methylation specific PCR (MSP primers) are represented by arrows. The coding start site is indicated by a bent arrow (+1). Solid squares indicate locations of exons. (B) The first and (C) second $\mathrm{CpG}$ islands are represented. The vertical lines mark the locations of $\mathrm{CpG}$ dinucleotide sites. Each row of circles represents the methylation pattern obtained from individual clones of the TUBB3 gene. Each circle represents a $\mathrm{CpG}$ dinucleotide site. The filled circles are methylation positive and the clear circles are methylation negative. Methylation is frequently observed in the second $\mathrm{CpG}$ island mRNA/ protein expression.

were inversely correlated with those of the acetylation status of the TUBB3 gene. Thus, these data suggest that DNA demethylation at the second $\mathrm{CpG}$ site is required for gain of TUBB3 protein overexpression, and that chromatin acetylation is a factor that accelerates TUBB3 $\mathrm{mRNA}$ /protein expression in ovarian cancer cell lines.

Methylation-specific PCR. Using the MSP method, we evaluated the promoter status at the TUBB3 gene in 14 primary ovarian cancers, and compared it with the results of immunostaining for TUBB3 protein. PCR was successful for the methylated-primer set (M) in all cases, and positive signals for the unmethylated-primer set (U) were observed in $10(71 \%)$ of the 14 tumors (Fig. 7). Although DNAs
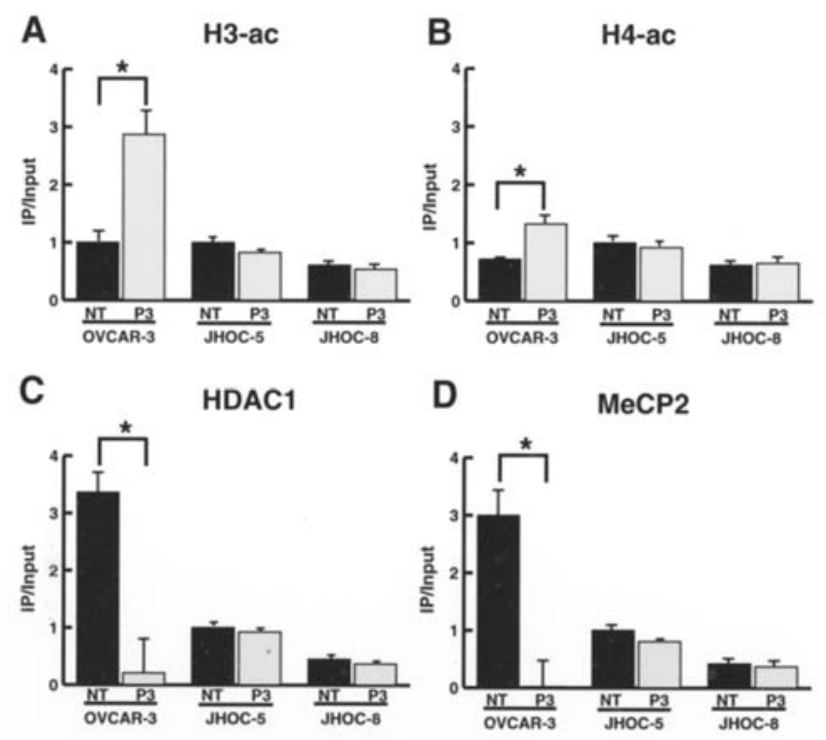

Figure 6. ChIP assays for acetylated-histone-H3 (A, H3-ac) and -H4 (B, H4ac), HCAD1 (C) and MeCP2 (D) in the region of the second CpG site. IP/Input (DNA immunoprecipitated with each antibody-non-specific antibody control [NAC])/input DNA-NAC). Values represent the mean $\pm \mathrm{SE}$ of 3 experiments from 2 independent ChIP assays. In OVCAR-3, acetylated $\mathrm{H} 3$ and $\mathrm{H} 4$ histones are increased after treatment with HDAC inhibitor (P3 and $3 \mathrm{mM}$ PBA). An inverse relationship to the results for actylated histones is evident for HDAC1 and MeCP2. NT, untreated control. ${ }^{*} \mathrm{P}<0.05$.

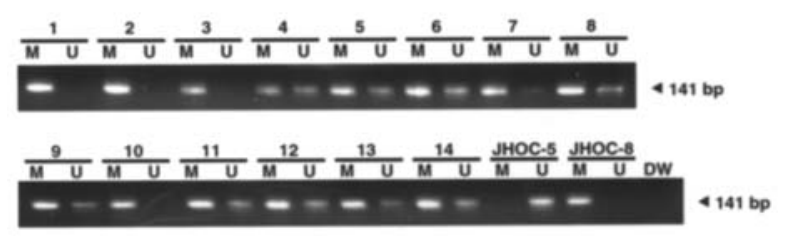

Figure 7. MSP analyses for the TUBB3 gene in 14 ovarian cancers. Numbers indicate the patient number. $M$, methylated primer, and $U$, ummethylated primer. Methylation status at the second $\mathrm{CpG}$ island (Fig. 5C) of the TUBB3 gene is determined by the presence of the signal using the unmethylated-primer set. Patient numbers 4, 5, 6, 7, 8, 9, 11, 12, 13 and 14 are judged to have the unmethylated type.

extracted from whole ovary are not appropriate for correct evaluation of the methylation status of the non-cancerous ovary, we preliminarily examined 10 non-cancerous ovarian tissues by the MSP method. No signal for the unmethylatedprimer set was found (data not shown). We assessed the presence or absence of methylation signals at the second CpG island of the TUBB3 gene using an unmethylatedprimer set. There was a tendency for an inverse correlation between DNA methylation status and TUBB3 protein expression, but this was not significant.

\section{Discussion}

Drug resistance represents a complex problem in the treatment of ovarian cancer. Patients with clear cell carcinoma (CCC), which is a special histological type of ovarian cancer with an incidence higher in Japan than in Western countries 
$(16 \%$ vs. $6-8 \%)(33,34)$, show a very low rate of response to platinum-based chemotherapy and a high incidence of progressive disease $(33,34)$. Immunohistochemistry for $\mathrm{ABCB} 1$ is a good biomarker for predicting drug resistance in ovarian CCCs $(35,36)$, and immunopositivity for TUBB3 protein is also a prognostic indicator in advanced ovarian cancers (23). The number of subjects in the present study was insufficient for evaluating the significance of TUBB3 immunostaining for the prediction of overall patient survival or tumor chemosensitivity because of the varied regimens we employed, and the short follow-up periods. However, some forms of aggressive tumor behavior (such as clinical stage, and the presence of ascites or lymph node metastasis) were frequently observed in patients with aberrant TUBB3 protein expression, in agreement with previous reports (23), whereas there was no significant relationship between histological type and aberrant expression of TUBB3 protein. Assessment of aberrant TUBB3 protein expression could be useful for identifying ovarian cancer patients with poor prognosis so that they can be given more aggressive and/or targeted therapy.

The present study is the first to have demonstrated that epigenetic mechanisms are partly involved in aberrant TUBB3 protein expression in ovarian cancer. The 5'-flanking region sequences and transcriptional regulation of the TUBB3 gene were first elucidated in the rat genome (37). The minimal region needed to drive expression of the TUBB3 gene contains multiple putative binding sites for the transcription factors SP1 and AP2, as well as a central nervous system enhancer regulatory element and an E-box (37). In addition, the first $490 \mathrm{bp}$ of the promoter are sufficient for regulating TUBB3 gene expression during neuronal differentiation in the murine embryonic carcinoma cell line PCC7 (37). Multiple putative binding sites for SP1 and AP2 have also been identified in promoter regions of the human TUBB3 gene, and the present study demonstrated that such sites were located within the first $\mathrm{CpG}$ island, which was hypomethylated in all the cell lines examined.

We searched for transcriptional signatures within the heavily methylated regions in the second $\mathrm{CpG}$ island, and identified the binding site of the zinc-finger gene-specific repressor element RE-1 silencing transcriptional silencing factor/neuronal restricted silencing factor (REST/NRSF) $(38,39)$. This transcription factor has been proposed to play an important role in establishing and maintaining the expression of neuron-specific genes by recruiting various chromatin-modifying enzymes such as class I HDACs (HDAC1 and HDAC2), Sin3, G9a, and CoREST (40-44). Moreover, REST was recently identified as a tumorsuppressor gene in epithelial cells (45). Deletions of the human chromosome on which REST is located are frequently observed in colon cancer, and loss of heterozygosity for REST has been identified in one-third of primary human colon cancers (45). In the same study, a frameshift mutation in REST resulting in a truncated protein that lacks the $\mathrm{C}$-terminal repression domain was identified in colon cancer cell lines examined (45). In addition, a splice variant of REST that lacks the DNA binding domain and the C-terminal repression domain is frequently observed in small cell lung cancers (46). These factors might be involved in aberrant
TUBB3 expression in diverse human malignancies (including colon and small cell lung cancers). Thus, REST might be a key molecule for investigating epigenetic gene regulation associated with tumor development and aggressive tumor behavior, including drug resistance, in human malignancies.

All of these previous observations are consistent with the role of REST as a global silencer of neuron-specific gene expression outside of the nervous system $(38,39)$. As our results for TUBB3 expression after treatment with demethylating agent and/or HDAC inhibitor were not always concordant with the classical concept of the expression mechanism of REST-targeting molecules, interpretation of our data is difficult. If the full length of the REST protein is expressed and acts as a transcriptional repressor, the TUBB3 gene would be repressed in the absence of DNA methylation, and a gain of TUBB3 expression would be induced by treatment with the HDAC inhibitor, PBA. Although the results for OVCAR-3 are compatible with the classical concept of REST-targeting molecules, other mechanisms have to be considered in the remaining cell lines.

Recent publications have described two regulatory mechanisms for REST-mediated gene silencing by transient repression and long-term silencing of specific genes in different tissues (47-50). During neural stem-cell differentiation, although REST binding is lost from RE-1 sequences, the CoREST complex remains bound and continues to mediate the repression of the associated genes (51). This continuous repression seems to be facilitated by DNA methylation and MeCP2 recruitment (51). DNA methylation of REST-targeting genes plays an important role in their expression in the long-term silencing mechanism $(48,51)$. It has remained unclear why treatment with demethylating agents induces a gain of TUBB3 protein expression but not downregulation. To resolve this contradiction, we will need to carry out structural and expression analyses of the REST gene (mutations, splice variants and protein expression level). Preliminarily, we have screened REST protein expression in human cancer cell lines including ovarian cancers, and in the REST-negative cell line JHOC-8 (unpublished data). Disruption of the long-term silencing mediated by DNA methylation and $\mathrm{MeCP} 2$ recruitment might be involved in the expression of TUBB3 protein. We are now planning to conduct a systematic examination of mutation/splice variants/ gene expression of the REST gene and their association with epigenetic status of the TUBB3 gene in diverse human malignancies with the aim of providing new insight into the underlying mechanism of taxane resistance.

\section{Acknowledgments}

This work was supported in part by Grants-in-Aid for Scientific Research (15591432, 16590290, and 16791346) from the Ministry of Education, Science, Sports and Culture of Japan, and the Open Translational Research Project, Advanced Medical Science Center, Iwate Medical University.

\section{References}

1. Landis SH, Murray T, Bolden S and Wingo PA: Cancer statistics. CA Cancer J Clin 49: 8-31, 1999. 
2. Montero A, Fossella F, Hortobagyi G and Valero V: Docetaxel for treatment of solid tumours: a systematic review of clinical data. Lancet Oncol 6: 229-239, 2005.

3. Herzog TJ and Pothuri B: Ovarian cancer: a focus on management of recurrent disease. Nat Clin Pract Oncol 3: 604-611, 2006.

4. Murata T, Haisa M, Uetsuka H, et al: Molecular mechanism of chemoresistance to cisplatin in ovarian cancer cell lines. Int J Mol Med 13: 865-868, 2004.

5. Vikhanskaya F, Siddique MM, Kei Lee M, Broggini M and Sabapathy K: Evaluation of the combined effect of p53 codon 72 polymorphism and hotspot mutations in response to anticancer drugs. Clin Cancer Res 11: 4348-4356, 2005

6. Kishi K, Doki Y, Yano M, et al: Reduced MLH1 expression after chemotherapy is an indicator for poor prognosis in esophageal cancers. Clin Cancer Res 9: 4368-4375, 2003.

7. Orr GA, Verdier-Pinard P, McDaid $\mathrm{H}$ and Horwitz SB: Mechanisms of Taxol resistance related to microtubules. Oncogene 22: 7280-7295, 2003.

8. Giannakakou P, Sackett DL, Kang YK, et al: Paclitaxelresistant human ovarian cancer cells have mutant beta-tubulins that exhibit impaired paclitaxel-driven polymerization. J Biol Chem 272: 17118-17125, 1997.

9. Monzo M, Rosell R, Sanchez JJ, et al: Paclitaxel resistance in non-small-cell lung cancer associated with beta-tubulin gene mutations. J Clin Oncol 17: 1786-1793, 1999.

10. Mesquita B, Veiga I, Pereira D, et al: No significant role for beta tubulin mutations and mismatch repair defects in ovarian cancer resistance to paclitaxel/cisplatin. BMC Cancer 5: 101, 2005.

11. Sale S, Oefner PJ and Sikic BI: Re: genetic analysis of the betatubulin gene, TUBB, in non-small-cell lung cancer. J Natl Cancer Inst 94: 776-7; author reply 7, 2002.

12. Lamendola DE, Duan Z, Penson RT, Oliva E and Seiden MV: beta tubulin mutations are rare in human ovarian carcinoma. Anticancer Res 23: 681-686, 2003.

13. Kavallaris M, Kuo DY, Burkhart CA, et al: Taxol-resistant epithelial ovarian tumors are associated with altered expression of specific beta-tubulin isotypes. J Clin Invest 100: 1282-1293, 1997.

14. Kavallaris M, Burkhart CA and Horwitz SB: Antisense oligonucleotides to class III beta-tubulin sensitize drug-resistant cells to Taxol. Br J Cancer 80: 1020-1025, 1999.

15. Ranganathan S, Benetatos CA, Colarusso PJ, Dexter DW and Hudes GR: Altered beta-tubulin isotype expression in paclitaxelresistant human prostate carcinoma cells. Br J Cancer 77: 562-566, 1998.

16. Derry WB, Wilson L, Khan IA, Luduena RF and Jordan MA: Taxol differentially modulates the dynamics of microtubules assembled from unfractionated and purified beta-tubulin isotypes. Biochemistry 36: 3554-3562, 1997.

17. Hari M, Yang H, Zeng C, Canizales M and Cabral F: Expression of class III beta-tubulin reduces microtubule assembly and confers resistance to paclitaxel. Cell Motil Cytoskeleton 56: 45-56, 2003.

18. Bernard-Marty C, Treilleux I, Dumontet C, et al: Microtubuleassociated parameters as predictive markers of docetaxel activity in advanced breast cancer patients: results of a pilot study. Clin Breast Cancer 3: 341-345, 2002.

19. Rosell R, Scagliotti G, Danenberg KD, et al: Transcripts in pretreatment biopsies from a three-arm randomized trial in metastatic non-small-cell lung cancer. Oncogene 22: 3548-3553, 2003.

20. Seve P, Mackey J, Isaac S, et al: Class III beta-tubulin expression in tumor cells predicts response and outcome in patients with non-small cell lung cancer receiving paclitaxel. Mol Cancer Ther 4: 2001-2007, 2005.

21. Mozzetti S, Ferlini C, Concolino P, et al: Class III beta-tubulin overexpression is a prominent mechanism of paclitaxel resistance in ovarian cancer patients. Clin Cancer Res 11: 298-305, 2005.

22. Luduena RF: Multiple forms of tubulin: different gene products and covalent modifications. Int Rev Cytol 178: 207-275, 1998.

23. Ferrandina G, Zannoni GF, Martinelli E, et al: Class III beta-tubulin overexpression is a marker of poor clinical outcome in advanced ovarian cancer patients. Clin Cancer Res 12: 2774-2779, 2006

24. Ohishi Y, Oda Y, Basaki Y, et al: Expression of beta-tubulin isotypes in human primary ovarian carcinoma. Gynecol Oncol 105: 586-592, 2007.
25. Gan PP, Pasquier E and Kavallaris M: Class III beta-tubulin mediates sensitivity to chemotherapeutic drugs in non-small cell lung cancer. Cancer Res 67: 9356-9363, 2007.

26. Lee KM, Cao D, Itami A, et al: Class III beta-tubulin, a marker of resistance to paclitaxel, is overexpressed in pancreatic ductal adenocarcinoma and intraepithelial neoplasia. Histopathology 51: 539-546, 2007.

27. Enokida H, Shiina H, Igawa M, et al: CpG hypermethylation of MDR1 gene contributes to the pathogenesis and progression of human prostate cancer. Cancer Res 64: 5956-5962, 2004.

28. Baker EK and El-Osta A: MDR1, chemotherapy and chromatin remodeling. Cancer Biol Ther 3: 819-824, 2004.

29. Saito Y, Liang G, Egger G, et al: Specific activation of microRNA-127 with downregulation of the proto-oncogene BCL6 by chromatin-modifying drugs in human cancer cells. Cancer Cell 9: 435-443, 2006.

30. Akiyama Y, Maesawa C, Ogasawara S, Terashima M and Masuda T: Cell-type-specific repression of the maspin gene is disrupted frequently by demethylation at the promoter region in gastric intestinal metaplasia and cancer cells. Am J Pathol 163: 1911-1919, 2003.

31. Ogasawara S, Maesawa C, Yamamoto M, et al: Disruption of cell-type-specific methylation at the Maspin gene promoter is frequently involved in undifferentiated thyroid cancers. Oncogene 23: 1117-1124, 2004.

32. Fujisawa K, Maesawa C, Sato R, et al: Epigenetic status and aberrant expression of the maspin gene in human hepato-biliary tract carcinomas. Lab Invest 85: 214-224, 2005.

33. Itamochi H, Kigawa J, Sugiyama T, Kikuchi Y, Suzuki M and Terakawa N: Low proliferation activity may be associated with chemoresistance in clear cell carcinoma of the ovary. Obstet Gynecol 100: 281-287, 2002.

34. Sugiyama T, Kamura T, Kigawa J, et al: Clinical characteristics of clear cell carcinoma of the ovary: a distinct histologic type with poor prognosis and resistance to platinum-based chemotherapy. Cancer 88: 2584-2589, 2000.

35. Yokoyama Y, Sato S, Fukushi Y, Sakamoto T, Futagami M and Saito Y: Significance of multi-drug-resistant proteins in predicting chemotherapy response and prognosis in epithelial ovarian cancer. J Obstet Gynaecol Res 25: 387-394, 1999.

36. Yakirevich E, Sabo E, Naroditsky I, Sova Y, Lavie O and Resnick MB: Multidrug resistance-related phenotype and apoptosis-related protein expression in ovarian serous carcinomas. Gynecol Oncol 100: 152-159, 2006.

37. Dennis K, Uittenbogaard M, Chiaramello A and Moody SA: Cloning and characterization of the 5'-flanking region of the rat neuron-specific Class III beta-tubulin gene. Gene 294: 269-277, 2002.

38. Chong JA, Tapia-Ramirez J, Kim S, et al: REST: a mammalian silencer protein that restricts sodium channel gene expression to neurons. Cell 80: 949-957, 1995.

39. Schoenherr CJ and Anderson DJ: The neuron-restrictive silencer factor (NRSF): a coordinate repressor of multiple neuron-specific genes. Science 267: 1360-1363, 1995.

40. Andres ME, Burger C, Peral-Rubio MJ, et al: CoREST: a functional corepressor required for regulation of neural-specific gene expression. Proc Natl Acad Sci USA 96: 9873-9878, 1999.

41. Grimes JA, Nielsen SJ, Battaglioli E, et al: The co-repressor $\mathrm{mSin} 3 \mathrm{~A}$ is a functional component of the REST-CoREST repressor complex. J Biol Chem 275: 9461-9467, 2000.

42. Huang Y, Myers SJ and Dingledine R: Transcriptional repression by REST: recruitment of Sin $3 \mathrm{~A}$ and histone deacetylase to neuronal genes. Nat Neurosci 2: 867-872, 1999.

43. Naruse Y, Aoki T, Kojima T and Mori N: Neural restrictive silencer factor recruits $\mathrm{mSin} 3$ and histone deacetylase complex to repress neuron-specific target genes. Proc Natl Acad Sci USA 96: 13691-13696, 1999.

44. Roopra A, Sharling L, Wood IC, et al: Transcriptional repression by neuron-restrictive silencer factor is mediated via the Sin3histone deacetylase complex. Mol Cell Biol 20: 2147-2157, 2000.

45. Westbrook TF, Martin ES, Schlabach MR, et al: A genetic screen for candidate tumor suppressors identifies REST. Cell 121: 837-848, 2005.

46. Coulson JM, Edgson JL, Woll PJ and Quinn JP: A splice variant of the neuron-restrictive silencer factor repressor is expressed in small cell lung cancer: a potential role in derepression of neuroendocrine genes and a useful clinical marker. Cancer Res 60: 1840-1844, 2000 . 
47. Lunyak VV, Burgess R, Prefontaine GG, et al: Corepressordependent silencing of chromosomal regions encoding neuronal genes. Science 298: 1747-1752, 2002.

48. Toshiyuki $\mathrm{N}$ and Ichiro $\mathrm{M}$ : Molecular mechanisms regulating cell type specific expression of BMP/RA inducible neuralspecific Protein-1 that suppresses cell cycle progression: roles of NRSF/REST and DNA methylation. Brain Res Mol Brain Res 125: 47-59, 2004.

49. Belyaev ND, Wood IC, Bruce AW, Street M, Trinh JB and Buckley NJ: Distinct RE-1 silencing transcription factorcontaining complexes interact with different target genes. J Biol Chem 279: 556-561, 2004.
50. Ooi L and Wood IC: Chromatin crosstalk in development and disease: lessons from REST. Nat Rev Genet 8: 544-554, 2007.

51. Chen ZF, Paquette AJ and Anderson DJ: NRSF/REST is required in vivo for repression of multiple neuronal target genes during embryogenesis. Nat Genet 20: 136-142, 1998. 